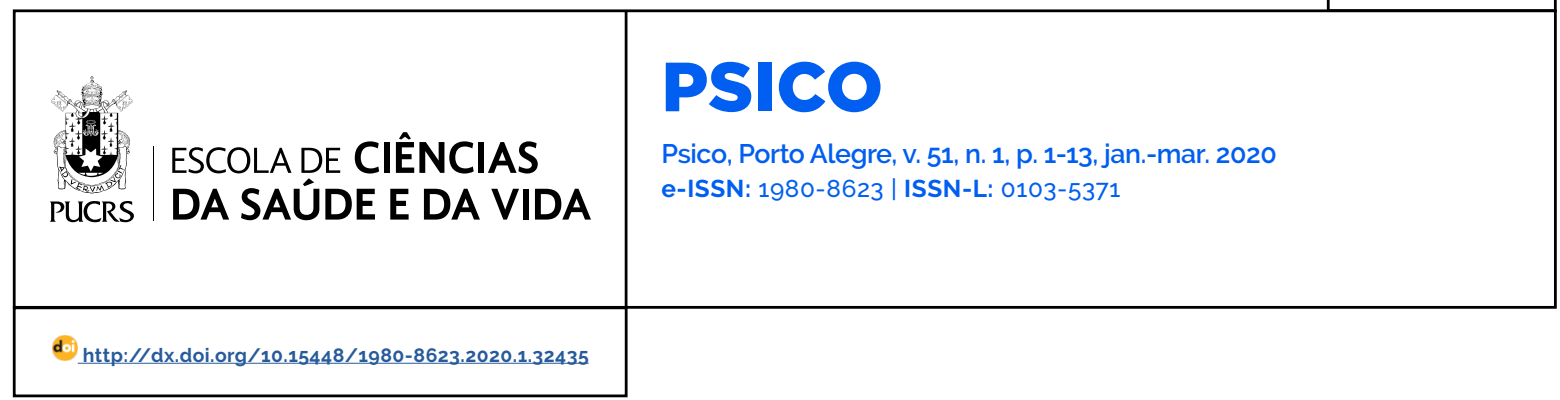

REVISÃO

\title{
Atendimento a vítimas de violência sexual: revisão da literatura acerca do treinamento de policiais
}

\author{
Supporting victims of sexual violence: a literature review on police officers' specialized training \\ Atención a las víctimas de violencia sexual: revisión de la literatura acerca del \\ entrenamiento policial
}

\section{Arielle Sagrillo \\ Scarpati ${ }^{1}$ \\ orcid.org/0000-0002-9817-6527 \\ silvia.koller@gmail.com}

Silvia Helena Koller ${ }^{1}$

orcid.org/0000-0001-9109-6674 arielle.psicologia@gmail.com

Recebido em: 4 nov. 2018 Aprovado em: 8 ago. 2019 Publicado em: $x x$ Xxx. 2020.
Resumo: Partindo-se do pressuposto de que a polícia desempenha papel fundamental no atendimento a casos de violência sexual, este estudo teve por objetivo explorar a existência de protocolos de treinamento contemplando técnicas de entrevista e de acolhimento de vitimas construidas com base em evidências científicas, bem como informações acerca de sua efetividade. Através de uma revisão sistemática da literatura levantaram-se trabalhos publicados entre 2009 e 2019, em quatro diferentes bases de dados: PubMed, Scientific Electronic Library Online (Scielo), Periódicos Eletrônicos de Psicologia (PePSIC) e Periódicos Capes. Os descritores empregados foram: "police training" AND (rape OR "sexual violence"). De 1.566 estudos encontrados, quatro atenderam aos critérios de inclusão e exclusão; todos esses publicados em língua inglesa. Coletivamente, os resultados evidenciam uma carência de materiais produzidos sobre o assunto, especialmente em contexto brasileiro, e alertam para a necessidade de mais diálogo entre a academia e a prática profissional. Limites e direcionamentos futuros também são apresentados

Palavras-chave: polícia, revisão de literatura, treinamento, violência sexual, vitima.

Abstract: Assuming that police officers play a key role in cases of sexual violence against women, this study aimed to explore the existence of standardized, evidence-based, practical guidelines and techniques for interviewing and helping victims of sexual violence, as well as its effectiveness. Using a Systematic Review of Literature, papers published between 2009 e 2019 were searched in four different databases: PubMed, Scientific Electronic Library Online (Scielo), Periódicos Eletrônicos de Psicologia (PePSIC) and CAPES Databank. The employed descriptors were: "police training" AND (rape OR "sexual violence"). Out of 1.566 studies, only four met the inclusion and exclusion criteria. The results show a lack of materials produced on the subject, especially in Brazil, and alert to need for a dialogue between academics and practitioners. Limitations and future directions are presented and discussed.

Keywords: police, literature review, training, sexual violence, victims.

Resumen: La policía desempeña un papel fundamental en la atención a casos de violencia contra las mujeres. Por eso, este estudio tuvo por objetivo explorar la existencia de orientaciones prácticas y técnicas (baseadas em evidencias científicas) estandarizadas de entrevista y acogida de victimas de violencia sexual; asi como su efectividad. A través de una Revisión Sistemática de la Literatura se buscaron trabajos publicados entre 2009 y diciembre 2019, en cuatro diferentes bases de datos: PubMed, Scientific Electronic Library Online (Scielo), Periódicos Eletrônicos de Psicologia (PePSIC) y CAPES Databank. Los descriptores empleados fueron: "police training" AND (rape OR "sexual violence"). De 1.566 estudios, solo cuatro cumplieron con los criterios de inclusión y exclusión. Los resultados muestran una falta de materiales producidos sobre el tema, especialmente en Brasil, y advierte sobre la necesidad de un diálogo entre académicos y profesionales. Se presentan y discuten las limitaciones y direcciones futuras.

Palabras clave: la policía, revisión de literatura, formación, violencia sexual, víctima.
Artigo está licenciado sob forma de uma licença Creative Commons Atribuicão 4.0 Internacional. 
Estimativas sugerem que uma em cada três mulheres em todo o mundo já foi (ou está sob o risco de ser) agredida sexualmente em algum momento de sua vida (ex., UNICEF, UN Women, UNFPA, ILO \& OSRSG/VAC, 2013). Especificamente no Brasil, dados apontam que apenas no ano de 2018, mais de 27 mil casos foram registrados por profissionais da saúde (Senado Federal, 2018). Um crescimento de $92.86 \%$ quando comparado ao ano de 2011; quando cerca de 14 mil casos de estupro a mulheres brasileiras foram registrados.

Coletivamente, esses dados sugerem que apesar dos consideráveis esforços de prevenção e erradicação, a violência sexual permanece como um grave problema de justiça e saúde pública (Resende, Urzedo, Oliveira, Segundo, \& Jorge, 2011; Scarpati, 2013; Scarpati \& Pina, 2017). Dadas as suas características, gravidade e, principalmente, alta incidência, essa forma de agressão chama a atenção de autoridades e pesquisadores ao redor do mundo; convidando-os a questionarem, dentre outras coisas, os fatores envolvidos em sua ocorrência e as medidas necessárias para combatê-la (Scarpati, 2013; Scarpati \& Pina, 2017).

Antes de prosseguir, é importante que se compreenda, no entanto, que mesmo quando provenientes de fontes confiáveis, estatísticas acerca desse fenômeno precisam ser vistas com cautela. Para autores como Cerqueira e Coelho (2014), Scarpati e Pina (2017) e Venema (2018), em razão de uma variedade de motivos bem documentados, cerca de 90\% das vítimas simplesmente não revela às autoridades competentes que sofreu violência sexual; o que torna complexa a tarefa de estimar, corretamente, a magnitude do problema.

Dentre os fatores que contribuem para que as vitimas silenciem a violência sofrida, encontram-se: a ameaça de vingança dos agressores, a vergonha, e até mesmo o sentimento de culpa (Faúndes, Rosas, Bedone, \& Orozco, 2006; Porter, 1992). Outros motivos frequentemente citados referemse a possivel divulgação do caso pela imprensa, o medo de represálias e a desconfiança com as instituições de segurança e justiça no Brasil (Bueno, Lima, Pinheiro, Astolfi, Santos, \& Hanashiro, 2016).
Especificamente a respeito deste último ponto, o que se sabe é que, apesar dos avanços resultantes da instalação das primeiras DEAMs (Delegacias de Atendimento à Mulher), ainda na década de 1980, e mais notadamente, desde a inauguração da Lei Maria da Penha, já nos anos 2000 (Brasil, 2006), ainda há muito a ser feito no que tange às medidas de enfrentamento à violência contra a mulher no Brasil. Especialmente, no que se refere às violações na esfera sexual.

Isso porque, apesar da criação de delegacias especializadas e da edição de algumas normas e medidas técnicas que visam regulamentar o atendimento oferecido a mulheres vítimas de violência (ex., a Lei n. ${ }^{\circ}$ 11.530/2007 e a Política Nacional de Enfrentamento à Violência contra as Mulheres/2011), pouco foi o investimento em capital humano (Foscarini, 2010); fazendo com que uma série de crenças (ex. mitos de estupro), tabus e disputas ideológicas inviabilizem o debate de questões fundamentais para o atendimento de vitimas de violência sexual e comprometam a qualidade do serviço prestado (Bueno et al., 2016; Murphy \& Hine, 2018). Além disso, diferentemente da área da Saúde, onde há uma ampla literatura discorrendo tanto sobre a necessidade de protocolos de atendimento quanto sobre a eficácia de iniciativas de capacitação de recursos humanos para prevenção e cuidado às vítimas de violência (Ministério da Saúde, 2012; Secretaria de Estado da Saúde do Paraná, 2015, 2018; Silva, 2017), o mesmo não se observa em outras em áreas, como a Segurança e a Justiça.

Sobre essa primeira área, em verdade, o que se vê, é uma polícia que apesar de conhecer as consequências negativas da violência sexual, ainda: a) interpreta inadequadamente o(s) comportamento(s) das vítimas durante o atendimento dos casos, b) não possui ciência dos efeitos (deletérios) que a sua abordagem pode invocar nas mesmas e, portanto, c) subestima os impactos de suas intervenções na progressão do caso (Venema, 2016; 2018).

Na prática, isso se traduz em constantes experiências de vitimização secundária por parte das vítimas e uma queda na probabilidade de 
que elas procurem ajuda (McMilla, 2015; Murphy \& Hine, 2018). Por isso, para autores como Bueno e colegas (2016), faz-se mister que o currículo de policiais seja revisto, de modo a contemplar as especificidades da violência sexual e incluir temais tais quais: o acolhimento das vitimas e os encaminhamentos necessários.

Acrescente-se a isso, a necessidade de uma melhor compreensão dos aspectos culturais que dão sustentação aos discursos de responsabilização das vítimas (Scarpati, 2013) e o reconhecimento de como crenças distorcidas, preconceituosas e/ou estereotipadas, acerca do estupro, suas vitimas e seus agressores (i.e., mitos de estupro) podem interferir no acolhimento adequado desse público (Scarpati, Guerra, \& Nogueira-Bonfim, 2014). Isso porque, é no relatório inicial e na entrevista policial subsequente que se obtém evidência de qualidade do caso e, invariavelmente, se determinam as decisões e as ações penais posteriores (McMillan, 2015). Por isso, um aspecto chave da resposta às violações e crimes sexuais consiste no reconhecimento do papel central desempenhado por policiais no processo investigativo e na ampliação do escopo de trabalho de pesquisadores e de profissionais; que deve abranger não apenas juizes e promotores (Venema, 2018), mas também, policiais.

Assim, tendo como base a relevância do tema exposto, e reconhecendo a necessidade de que as críticas à polícia se traduzam no desenvolvimento de ações que permitam a superação deste problema (McMillan, 2015). este trabalho objetiva identificar a existência de protocolos de treinamento de policiais para entrevista e acolhimento de vítimas, bem como informações acerca de sua efetividade. Para isso, serão apresentados os resultados de uma revisão sistemática da literatura (Zoltowski, Costa, Teixeira, \& Koller, 2014).

\section{Método}

Este estudo constitui-se em uma revisão sistemática da literatura que, seguindo o modelo proposto por Zoltowski, Costa, Teixeira e Koller (2014) foi realizada por meio das seguintes etapas: a) formulação e delimitação do problema de pesquisa; b) delimitação das bases de dados a serem utilizadas; c) eleição dos termos de busca e dos operadores booleanos; d) busca e organização dos resultados; e) aplicação dos critérios de inclusão e exclusão definidos; f) extração dos dados selecionados; g) avaliação dos artigos; e, por fim, h) organização e interpretação dos dados.

A busca foi realizada por dois juizes independentes, e considerou os trabalhos publicados entre os anos de 2009 e 2019 . configurando, portanto, os dez últimos anos de produção científica acerca da temática escolhida. As bases de dados utilizadas foram: PubMed, Scientific Electronic Library Online (SciELo), Periódicos Eletrônicos de Psicologia (PePSIC) e Periódicos Capes. Como termos de busca, foram aplicados: "police training", rape e "sexual violence". Mais precisamente, na forma da expressão: "police training" AND (rape OR "sexual violence").

Foram adotados como critérios de inclusão/ exclusão: o a) tipo de documento; o b) idioma; o c) ano de publicação; e os d) objetivos do trabalho. Nesse sentido, foram incluidos, apenas: a) artigos publicados em periódicos revisados por pares; b) produzidos em português e/ou inglês; c) entre os anos de 2009 e 2019; e d) que apresentassem e/ou avaliassem protocolos de treinamento para policiais que contemplassem técnicas de entrevista e de acolhimento de vítimas. Tendo sido aplicados os critérios mencionados, prosseguiuse com a exclusão de artigos duplicados e a leitura de seus títulos, resumos e palavras-chave. Por fim, aqueles que atendiam ao objetivo desta revisão foram selecionados para leitura completa, análise e interpretação de seus resultados.

\section{Resultados}

Dos 1.566 artigos encontrados, apenas quatro foram considerados nesta revisão. Estes foram codificados em Artigo 01, Artigo 02, Artigo 03 e Artigo 04 e serão apresentados a seguir. A Figura 1 sintetiza as fases de seleção deste material. 0 ano de publicação, o país de origem do estudo, a metodologia utilizada e o perfil dos participantes foram analisados estatisticamente, de forma 
descritiva, por meio de frequências e porcentagens. Uma descrição geral dos artigos selecionados (objetivos, metodologia dos protocolos e resultados) encontra-se disposta na Tabela 1.

\begin{tabular}{|c|c|c|c|}
\hline \multicolumn{4}{|c|}{$\begin{array}{l}\text { Etapa } 1 \\
\text { Estudos identificados a partir da busca nas seguintes bas }\end{array}$} \\
\hline $\begin{array}{l}\text { PubMed } \\
(\mathrm{n}=1)\end{array}$ & $\begin{array}{l}\text { Scientific Electronic } \\
\text { Library Online (SciELo) } \\
(\mathrm{n}=23)\end{array}$ & $\begin{array}{l}\text { Periódicos Eletrônicos de } \\
\text { Psicologia (PePSIC) } \\
(\mathrm{n}=0)\end{array}$ & $\begin{array}{l}\text { Periódicos Capes } \\
\quad(\mathrm{n}=1.542)\end{array}$ \\
\hline \multicolumn{4}{|c|}{$\begin{array}{l}\text { Total de artigos selecionados } \\
\qquad(\mathrm{n}=1.566)\end{array}$} \\
\hline \multicolumn{4}{|c|}{$\begin{array}{c}\text { Etapa } 2 \\
\text { Exclusão de conteúdo duplicado e aplicação dos critérios de inclusão/exclusão } \\
(\mathrm{n}=200)\end{array}$} \\
\hline \multicolumn{4}{|c|}{$\begin{array}{l}\text { Etapa } 3 \\
\text { Leitura dos títulos, palavras-chave e resumo dos trabalhos selecionados na Etapa } 2 \\
(\mathrm{n}=43)\end{array}$} \\
\hline \multicolumn{4}{|c|}{$\begin{array}{c}\text { Etapa } 4 \\
\text { Leitura completa dos trabalhos selecionados na Etapa } 3 \\
(\mathrm{n}=12)\end{array}$} \\
\hline \multicolumn{4}{|c|}{$\begin{array}{c}\text { Etapa } 5 \\
\text { Amostra final de artigos incluídos na revisão (avaliação, síntese e interpretação dos dados) } \\
(\mathrm{n}=4)\end{array}$} \\
\hline
\end{tabular}

Figura 01 1. Resultados da revisão sistemática nas bases de dados Scielo.org, PubMed, PpSIC.org e Portal Capes. Gráfico de autoria própria

Primeiramente, chama atenção o fato de que não foram encontrados, na literatura nacional e internacional, trabalhos publicados em português e/ou que contemplassem a realidade brasileira e atendessem aos critérios estabelecidos. Assim, os países cujas realidades foram retratadas nestes trabalhos foram: Estados Unidos (2), Libéria (1) e Austrália (1). O delineamento utilizado nos estudos foi majoritariamente quali-quantitativo e a distribuição do ano de publicação identificada foi a seguinte: 2013, 2014, 2015 e 2019. Indicando que, após alguns trabalhos iniciais sobre este assunto (ex. Lonsway et al., 2001; Patterson, 2004), seguiu-se um hiato de publicações, e mais recentemente houve uma retomada no interesse sobre a temática.

Por fim, no que se refere aos objetivos propostos, foi identificado que, com exceção de um trabalho (Darwinkel, Powell, \& Tidmarsh, 2013; que oferece uma breve descrição de uma proposta de treinamento), os demais (Bacon, 2015: Holland, \& Rabelo, 2014 e Venema,
Lorenz, \& Sweda, 2019) se dedicam à avaliação de treinamentos já são ofertados a policiais e militares por órgãos governamentais. Todos discutem as implicações dessas propostas para a prática profissional e fornecem orientações para futuros cursos e treinamentos.

\section{Sobre a avaliação de protocolos de treinamento já existentes}

A primeira categoria reúne os trabalhos que avaliam os protocolos de treinamento já existentes e que, apesar de não os descreverem com riqueza de detalhes, exploram as percepções dos participantes sobre esses aspectos e acabam por fornecer informações importantes para aqueles que objetivam implementar iniciativas similares (ex. pontos a serem melhorados, sugestões etc.). Além disso, oferecem indicios de quais foram os temas trabalhados, a metodologia utilizada e, principalmente, quais objetivos foram alcançados.

No Artigo 01, Holland e Rabelo (2014), por exemplo, dedicam-se à avaliação de um 
treinamento implementado em contexto norteamericano, como parte do programa obrigatório conduzido desde 2005 pelo SAPRO (Sexual Assault Prevention and Response Office). Através de análise secundária dos dados gerados pela "Pesquisa de Trabalho e Relações de Gênero dos Membros do Active Duty de 2010" (WGRA), concluem que a exposição de militares à treinamentos voltados para a questão da violação sexual é capaz de predizer não apenas um maior nivel de conhecimento dos procedimentos e condutas a serem adotados frente a esse fenômeno, mas também à redução na incidência de casos de violência sexual entre membros desta própria categoria.

Os autores ressaltam, no entanto, que para que isso seja possivel, é necessário que o conteúdo ofertado, e que o tempo de exposição ao treinamento sejam considerados suficientes pelos próprios participantes. Isso porque, de acordo com esses autores, os participantes que relataram exposição mínima ao conteúdo obrigatório, demonstram os mesmos níveis de domínio de informação daqueles que não receberam treinamento algum; sugerindo, pois, que uma capacitação deficiente pode ser tão problemática quanto a sua "não oferta" (Holland \& Rabelo, 2014).

Percurso semelhante foi traçado no Artigo 02, por Venema, Lorenz e Sweda (2019); cujo estudo objetivou descrever a percepção de policiais americanos acerca dos protocolos de treinamento aos quais foram expostos ao longo de suas carreiras. Para isso, foram realizadas perguntas a respeito da utilidade percebida do treinamento, das necessidades e prioridades de temáticas, e da crença na relação entre o treinamento ofertado e a condução de melhores investigações de agressão sexual. Por fim, foi questionado o interesse em treinamentos futuros. A partir do contato com 231 investigadores de agressão sexual, distribuidos em 174 agências policiais todo o estado de Illinois, os autores observaram que muitos destes profissionais não haviam recebido, ao longo de sua formação, esclarecimentos e orientações acerca de assuntos tradicionalmente considerados pela literatura como de suma importância para o trato desta temática (ex. Powell \& Cauchi, 2011; Scarpati, 2013; Temkin, 1999).

Mais precisamente, quase metade dos investigadores reportou não ter recebido formação sobre aceitação do mito de estupro ( $N=101$; $49 \%$ ), nem mesmo a respeito de como interagir com vítimas que sofrem de TEPT (Transtorno de Estresse Pós-Traumático) e/ou outras formas de traumas ( $N=91 ; 44 \%$ ). Cerca de um terço dos investigadores também relatou não receber nenhum treinamento em empatia ( $n=74 ; 36 \%$ ) e $42 \%$ não tiveram acesso a informações sobre como se portar frente vítimas de agressão sexual por parceiros íntimos ( $N=87$ ). Além disso, apenas $13 \%$ dos entrevistados recebeu informações sobre como conduzir entrevistas com as vitimas $(N=26)$.

Contrariamente, pôde-se observar que poucos foram aqueles que não receberam treinamento sobre as leis de agressão sexual ( $N=23 ; 11 \%)$, as motivações do agressor ( $N=34 ; 16 \%)$, e os procedimentos de informação ( $N=39 ; 19 \%$ ) e interação mais gerais durante o processo legal e encaminhamentos de serviço $(\mathrm{N}=34$; $16 \%$ ), por exemplo. O que indica que o foco do treinamento ainda está centrado nos aspectos mais burocráticos e práticos da rotina policial, em detrimento do desenvolvimento de habilidades e competências pessoais.

No que se refere à utilidade da formação recebida, a maioria dos itens propostos foi classificada como "minimamente útil", sendo o treinamento em técnicas para interrogar suspeitos considerado o mais útil $(M=3.52, D P=0,70)$ e o treinamento em interação com vítimas $(M=2,60$, $\mathrm{DP}=0,99)$ um dos menos úteis, sendo que as escalas variavam entre 1 e 5 . Importante destacar que esses resultados não foram explicados por variáveis como a experiência do funcionário e/ ou características demográficas. Contrariando a hipótese inicial dos autores. Por fim, participantes indicaram que treinamentos adicionais $(M=3,04$, DP $=1,00)$ e continuados $(M=2,92, \mathrm{DP}=0,92)$ poderiam levar a melhores investigações de agressão sexual. Especialmente no que se refere a temas como a identificação e a preservação de evidências $(M=3,75, D P=0,49)$, técnicas de entrevista $(M=$ 
3,66, SD = 0,54) que incluam informações sobre empatia, compaixão e respeito e orientações sobre a neurobiologia do trauma e a cultura do estupro (Venema, Lorenz, \& Sweda, 2019).

Em seguida, o Artigo 03 (Bacon, 2015;) consiste em um estudo de caso que avalia os esforços realizados pela Polícia Nacional da Libéria (LNP) para o recrutamento e o treinamento de oficiais mulheres em uma unidade especializada no atendimento de vítimas de violência sexual e de gênero (SGBV). De acordo com Bacon (2015), a preocupação central dessa iniciativa consistiu na capacitação de policiais para elaboração de relatórios investigativos, coleta de evidências e manutenção de confidencialidade em casos de denúncias por crimes de violência doméstica e agressão sexual. Destaca-se, frente aos demais, por ser o único trabalho a retratar a realidade de um país subdesenvolvido e por evidenciar a importância de um alinhamento entre aspectos como vontade política, calendário e contexto favoráveis à implementação de tais ações.

Para a avaliação dos resultados, esta análise se concentrou nas métricas de representação e responsividade. E, assim, uma vez que pode ser observado um acréscimo tanto no número de casos atendidos pela Seção de Proteção à Mulher e à Criança, quanto no número de mulheres policiais capacitadas para atuar nesses espaços, sugere-se o sucesso da iniciativa. Apesar desses indicativos, Bacon (2015) pondera que a sustentabilidade e o impacto global das reformas foram severamente prejudicados pela baixa capacidade técnica e pelo fraco Estado de Direito, e recomenda cuidado na interpretação dos dados; uma vez que avaliações externas da resposta da polícia à SGBV são mistas e há críticas ao modelo, assim como pouca ou nenhuma informação a respeito da maneira como essa avaliação foi realizada. Além disso, não há, nesse relato, informações a respeito de como esse treinamento foi conduzido.

\section{Sobre a criação e implementação de protocolos de treinamento}

Um único trabalho, representado pelo
Artigo 04 (Darwinkel, Powell, \& Tidmarsh, 2013), contemplando a criação e a implementação de protocolos de treinamento de policiais para atendimento de vítimas de violência sexual foi encontrado. Nele, os autores ampliam a discussão iniciada por Lonsway et al. (2001) e Patterson (2004) e testam a eficácia de um programa de treinamento obrigatório, projetado para policiais norte-americanos especializados em abuso sexual (infantil e adulto). O treinamento em questão, construido com base na abordagem "Whole Story" para a investigação de crimes sexuais, foi conduzido por profissionais treinados para tal função ao longo de quatro semanas. Esta abordagem, criada por Tidmarsh, Powell e Darwinkel (2012) centra-se na dinâmica da violência e em técnicas de entrevista, e tem como premissa teórica a ideia de que a violência acontece a partir da manipulação do comportamento da vítima, pelo agressor (especialmente em casos de violência sexual infantil).

Para além de aulas teóricas expositivas a respeito do comportamento das vítimas e dos agressores, são promovidas diversas situações interativas e role-plays, onde os participantes são solicitados a demonstrarem os seus conhecimentos e as suas habilidades de entrevista. Mais precisamente, esse treinamento está organizado da seguinte forma: modelo introdutório, compreensão do comportamento das vítimas (ex. respostas ao trauma, a psicologia das vítimas e o seu comportamento) e dos agressores (ex. motivações e perspectivas), discussão de casos e, por fim, técnicas de entrevista (teoria e prática).

Para o estudo, adotou-se um desenho pré e pós-intervenção, com todos os participantes completando um questionário que avaliava a sua percepção dos casos no primeiro e no último do treinamento. Os questionários aplicados incluiam 12 cenários, seguidos de uma série de quatro perguntas: a) "Até que ponto você está confiante de que o caso será julgado?" (nem um pouco confiante/ extremamente confiante); e b) "Quanta responsabilidade deve ser atribuida à vítima neste cenário?" (nenhuma responsabilidade/ toda responsabilidade). A pergunta final pediu 
aos policiais que justificassem a sua classificação sobre a autorização de casos, listando até cinco fatores (em ordem de importância) considerados relevantes por eles durante a tomada de decisões.

Participaram do treinamento, 77 oficiais da Policia de Victoria (Austrália). A amostra foi heterogênea (policiais regionais e metropolitanos), sendo majoritariamente composta por oficiais do sexo masculino ( $N=51 ; 66 \%$ ). Com relação à experiência prévia, essa variou de nenhuma a 20 anos (ou mais). Os resultados encontrados indicam mudanças nos níveis de confiança no relato da vitima e na responsabilidade atribuída à mesma. Mais precisamente, que a confiança no relato da vítima aumentou (do pré para o pós-intervenção), enquanto a percepção de sua "responsabilidade" sobre o ocorrido, diminuiu. Resultados apresentados também oferecem evidência qualitativa para justificar a importância de um treinamento focado na compreensão da dinâmica da violência sexual no processo de denúncia e de condução do caso. Um efeito adicional encontrado refere-se ao fato de que a probabilidade de instauração de inquérito foi maior pós-intervenção, particularmente nos casos em que a evidência em relação ao crime era ambígua (ou seja, casos em que os policiais não haviam atribuido veracidade inicial à declaração da vítima). Assim, é possivel sugerir que a exposição a um tipo de treinamento focado nos aspectos dinâmicos da violência sexual é capaz de reverter discursos de responsabilização da vítima.

\section{Discussão}

A violência sexual cometida contra mulheres é uma realidade no Brasil e no mundo. Considerado um problema de saúde e de segurança pública, essa forma de agressão atinge mulheres com diferentes perfis e em diversas localidades; desafiando profissionais no que tange à tarefa de prevenir a sua ocorrência e "remediar" os seus efeitos. Dados sugerem, por exemplo, que apesar dos esforços empregados por diferentes atores sociais e dos avanços alcançados (ex. legislações e políticas públicas), ainda são muitos os desafios a serem superados (Brasil, 2015). Um deles, especialmente, refere-se ao atendimento prestado por profissionais de segurança pública no momento da (ou mesmo após) denúncia do ocorrido.

Nesse contexto, e partindo-se do principio de que a polícia exerce papel fundamental no desfecho de casos de violência sexual (Powell \& Cashi, 2011; Taylor \& Gassner, 2010; Venema, 2016), o primeiro, e talvez mais importante, resultado encontrado consiste no fato de que não puderam ser identificados quaisquer trabalhos publicados em lingua portuguesa ou mesmo, retratando a realidade brasileira, que respondessem ao objetivo desta pesquisa. Além disso, mesmo quando consideradas as iniciativas internacionais, poucos foram os estudos publicados nesta última década.

Esse dado denuncia a lacuna, ainda presente, na literatura sobre o tema e sugere que, apesar da existência de uma preocupação com a reflexão sobre as consequências (negativas) desta forma de violência (Brown, 2011), essa não se traduz em estudos científicos e medidas práticas/efetivas de ação. O que difere da relevância científica encontrada em áreas de atendimento de casos de violência sexual na área da Saúde (Brasil, 2015; Pasinato, 2012), casos de violência doméstica e, ainda, violações contra crianças e adolescentes (Brasil, 2005; Oehme, Prost, \& Saunders, 2016; Powell, 2008) ao longo dos anos.

Como pontuado por autores como Brown (2011) e Silva (2017), apesar dos avanços realizados, ainda é longo o caminho a ser trilhado para um serviço de proteção capaz de "proporcionar a eficiente persecução criminal e responsabilização dos agressores sexuais" (Silva, 2017, p. 78). Além da ausência de um treinamento exclusivamente pensado para preparar as policias civil e militar para o acolhimento de vitimas de violência sexual, a limitação de recursos destinados a essa área, o desconhecimento da rede e a limitada quantidade de profissionais alocados nessa área torna ainda mais complexa essa tarefa.

Pensando-se no atual cenário, especificamente, no conteúdo a ser trabalhando com esses policiais, a revisão da literatura corrente não apenas permite algumas reflexões importantes, como também oferece importantes direcionamentos. 
Primeiramente, indica a necessidade de um maior investimento no desenvolvimento das habilidades humanas exigidas para o contato com individuos vitimados sexualmente. Conforme discutido por autores como Powell e Cauchi (2011) e mais recentemente, Venema, Lorenz e Sweda (2019), muitos são os fatores que atravessam (direta e indiretamente) a condução das entrevistas e o acolhimento de vítimas de violência sexual. Dentre esses fatores, destacam-se: o preconceito contra mulheres e as atitudes a respeito de temas como sexo e poder; e crenças estereotipadas acerca dessa forma de agressão (i.e., mitos de estupro); que têm ganhado um contorno mais sofisticado e sutil com os passar dos anos (Page, 2010; Murphy \& Hine, 2018).

Por isso, o trabalho com esse público exige profissionais capacitados em níveis técnicos (ex., consequências físicas e psicológicas da violência sexual, perfil do agressor e técnicas de entrevista) e pessoais (ex. habilidades sociais, atitudes e crenças frente ao fenômeno), por assim dizer (Campbell, Adams, Wasco, Ahrens, \& Sefl, 2009). Corroborando o exposto, Rich e Seffrin (2012) apresentam evidências de que uma mudança de atitude por parte dos policiais está significativamente $(p<.05)$ relacionada a uma melhora nas habilidades de entrevista com vítimas, por exemplo. Importante salientar que os benefícios desse tipo de capacitação não se resumem apenas a uma melhor qualidade no contato inicial entre vítimas e policiais, mas têm efeitos diretos na qualidade das provas obtidas e no andamento do caso. Facilitando, nesse sentido, a atuação deste profissional.

O desafio, no entanto, consiste em superar os desafios práticos relacionados ao desenvolvimento e à implementação de protocolos de treinamento que sejam capazes de abarcar, adequadamente, os aspectos supracitados. Lonsway, Welch e Fitzgerald (2001) e Patterson (2004), por exemplo, ao ofertarem treinamentos de curta duração foram eficazes na melhoria do desempenho comportamental em diversas habilidades práticas (incluindo entrevistas), mas não tiveram qualquer efeito sobre a aceitação dos mitos de estupro entre policiais.
Sendo esta informação de considerável valor, uma vez que os mitos de estupro estão associados à percepção de que a vítima (e nāo o agressor) é a pessoa responsável pela violência perpetrada e, como resultado, acabam por justificar a agressão cometida, ao mesmo tempo em que minimizam os danos gerados (Murphy \& Hine, 2018; Scarpati, Guerra, \& Nogueira-Bonfim, 2014). Alguns exemplos de mitos fazem referência a ideias como: "mulheres inventam que foram estupradas para se vingar de antigos companheiros"; "estupradores são pessoas esquisitas, psicóticas e/ou solitárias"; "estupros não acontecem dentro de relacionamentos", dentre outros (Scarpati, 2013). Essas matrizes de processos sociocognitivos, que sustentam as mudanças de atitude em ambientes informacionais, são complexas e exigem esforços ativos (e constantes) de seus participantes para que sejam alteradas (Wood, 2000). Assim, uma estratégia possivel consiste na construção de propostas de treinamentos de maior duração, tal como ofertado por Darwinkel, Powell e Tidmarsh (2013), em que o treinamento teve duração de quatro semanas.

Por fim, outro elemento importante, evidenciado pelos trabalhos supracitados, diz respeito à necessidade de um treinamento continuado, ao longo da carreira, e não apenas no momento de ingresso na corporação. Isso se torna relevante, uma vez que a constante exposição a cenários de agressões sexuais durante a sua carreira pode, progressivamente, dessensibilizar esse profissional frente aos aspectos traumáticos envolvidos na experiência da vítima (Venema, 2018). Em resumo, os dados aqui reunidos questionam os resultados contraditórios encontrados no berço desta linha de investigação (Ask, 2010; Campbell, 2005; Lonsway, Welch, \& Fitzgerald, 2001; Patterson, 2004; Sleath \& Bull, 2012) e indicam que treinamentos especializados para o atendimento de vítimas de violência sexual estão associados à uma melhoria na qualidade desse serviço. Para que isso seja possivel, no entanto, esses treinamentos devem ser planejados adequadamente. Ou seja, devem considerar aspectos tais quais: a) o tipo de 
conteúdo ministrado (ex., efeitos do trauma, mitos de estupro e outros vieses cognitivos); b) o tempo de exposição ao mesmo (predileção por programas de maior duração); e c) as técnicas utilizadas para tal (equilibrio entre teoria e prática).

\section{Conclusāo}

Este trabalho apresenta um panorama do estado da arte na literatura nacional e internacional acerca da existência de orientações práticas e técnicas padronizadas de entrevista e de acolhimento de vítimas de violência sexual por policiais. Para tal, foi realizada uma revisão sistemática da literatura (Zoltowski, Costa, Teixeira, \& Koller, 2014) de artigos revisados por pares, publicados nos últimos dez anos em periódicos nacionais e internacionais, e disponibilizados em quatro diferentes bases de dados.

Os resultados encontrados revelam a carência de publicações abordando essa temática; especialmente no Brasil. Chamam atenção, ainda, para a insuficiência de produções baseadas em evidências que permitam uma formação adequada de profissionais da área da segurança pública (Silva, 2017). Especialmente quando comparado a outras áreas. Por exemplo, a Saúde (ex., Brasil, 2015; Pasinato, 2012; Souza \& Adesse, 2005). Acredita-se, pois, que esse cenário contribua para uma formação profissional que, ao carecer de fundamentação e critérios rigorosos de construção, se traduza em uma assistência fragmentada, orientada por práticas e discursos de senso comum, e atravessada por crenças e atitudes pessoais, em detrimento de uma escuta qualificada e sensivel (Balbueno, 2011).

Para além de uma crítica ao modelo existente de treinamento, faz-se mister destacar que os trabalhos apresentados contribuem, de maneira importante, na identificação de algumas das variáveis relacionadas à (in)eficácia e à (in) efetividade de protocolos já implementados. Por conseguinte, também fornecem dados importantes para futuras iniciativas. Nesse sentido, reconhecendo que este é um campo a ser explorado nos centros de pesquisa do Brasil e do mundo, sugere-se que pesquisadores e profissionais se utilizem destes resultados para construção de materiais para consulta e didáticos, bem como protocolos de treinamento e educação permanente, especialmente pensados para atender a esta demanda. Recomenda-se, ainda, que profissionais já atuantes nesta área relatem as suas intervenções com o rigor científico necessário para que se inicie um movimento de reconhecimento e de validação destas propostas.

Finalmente, sobre as limitações deste trabalho, é preciso pontuar que a avaliação do material foi realizada pelas autoras, de maneira independente, e que não foram utilizados juízes externos na busca e análise dos materiais encontrados. Em caso de divergência na comparação dos resultados, buscou-se o consenso. Além disso, em função dos critérios de inclusão e exclusão adotados, e da utilização de apenas quatro bases de dados, é possivel que as buscas realizadas possam ter limitado o número de resultados.

Essas limitações não invalidam, no entanto, os resultados encontrados ou mesmo comprometem a relevância do estudo. Principalmente por ser esse, o primeiro trabalho desta natureza a ser conduzido em contexto brasileiro. Por isso, incentiva-se que estudos futuros se dediquem à expansão do referencial investigado. Seja através da inclusão de outros termos além daqueles utilizados, da inclusão de outros bancos de dados (ex., PsycINFO, ScienceDirect), ou mesmo de outros materiais; tais como relatos de práticas e/ ou documentos oficiais possam ampliar o escopo de compreensão do problema.

\section{Referências}

Ask, K. (2010). A survey of police officers' and prosecutors' beliefs about crime victim behaviors. Journal of Interpersonal Violence, 25(6), 1132-1149. https://doi. org/10.1177/0886260509340535

Bacon, L. (2015). Liberia's Gender-Sensitive Police Reform: Improving Representation and Responsiveness in a Post-Conflict Setting. International Peacekeeping, 22(4), 372-397. https://doi.org/10.1080/13 533312.2015.1059285.

Balbueno, B. (2011) Investigação sobre atendimento psicossocial oferecido em delegacias de defesa da mulher. Psicólogo inFormação, 15(15), 69-82. https:// doi.org/10.15603/2176-0.969/pi.v15n15p69-82 
Brasil (2005). Prevenção e tratamento dos agravos resultantes da violência sexual contra mulheres e adolescentes: norma técnica. 2. ed. Brasilia: Ministério da Saúde, Secretaria de Politicas de Saúde. Departamento de Gestão de Políticas Estratégicas. Área Técnica Saúde da Mulher. (DOI INEXISTENTE)

Brasil (2011). Politica Nacional de Enfrentamento à Violência contra as Mulheres. Brasilia, DF. (DOI INEXISTENTE)

Brown, J. (2011) We mind and we care but have things changed? Assessment of progress in the reporting, investigating and prosecution of allegations of rape. Journal of Sexual Aggression, 17(3), 263-272. https://doi.org/10.1080/13552600.2011.613280

Bueno, S., Lima, R. S. de, Pinheiro, M., Astolfi, R., Santos, T. \& Hanashiro, O. (2016). A Policia precisa falar sobre estupro: percepção sobre violência sexual e atendimento a mulheres vitimas de estupro nas instituições policiais. Fórum Brasileiro de Segurança Pública. 24pp. (DOI INEXISTENTE)

Campbell, R. (2005). What really happened? A validation study of rape survivors' help-seeking experiences with the legal and medical systems. Violence and Victims, 20, 55-68. https://doi.org/10.1891/vivi.2005.20.1.55

Campbell, R., Adams, A. E., Wasco, S. M., Ahrens, C. E. \& Sefl, T. (2009). Training interviewers for research on sexual violence: a qualitative study of rape survivors' recommendations for interview practice. Violence Against Women, 15(5), 595-617. https://doi. org/10.1177/1077801208331248

Cerqueira, D., \& Coelho, D. S. C. (2014). Estupro no Brasil: Uma radiografia segundo os dados da saúde (versão preliminar). Nota técnica, n.11, IPEA. (DOI INEXISTENTE)

Darwinkel, E., Powell, M. \& Tidmarsh, P. (2013) Improving police officers' perceptions of sexual offending through intensive training. Criminal Justice and Behaviour, 40(8), 895-908 (**)https://doi. org/10.1177/0093854813475348

Faúndes, A., Rosas, C. F., Bedone, A. J., \& Orozco, L. T. (2006). Violência sexual: Procedimentos indicados e seus resultados no atendimento de urgência de mulheres vitimas de estupro. Revista Brasileira de Ginecologia e Obstetricia, 28, 126-135. https://doi. org/10.1590/S0100-72032006000200009

Foscarini, L. T. (2010). As misérias do inquérito policial: a produção da investigação criminal na cidade de Porto Alegre - RS. Dissertação de Mestrado. Programa de Pós-Graduação em Ciências Criminais, PUCRS. Porto Alegre. (DOI INEXISTENTE)

Holland, K. J., Rabelo, V. C., \& Cortina, L. M. (2014). Sexual Assault Training in the Military: Evaluating Efforts to End the "Invisible War." American Journal of Community Psychology, 54(3-4), 289-303 https://doi. org/10.1007/s10464-014-9672-0

Lonsway, K. A., Welch, S., \& Fitzgerald, L. F. (2001) Police training in sexual assault response: Process, outcomes, and elements of change. Criminal Justice and Behavior, 28, 695-730. https://doi. org/10.1177/009385480102800602
McMillan, L. (2015) The role of the specially trained officer in rape and sexual offence cases. Policing and Society, 25(6), 622-640, https://doi.org/10.1080/1043 9463.2014 .912648

Ministério da Saúde (2012). Prevenção e Tratamento dos Agravos Resultantes da Violência Sexual contra Mulheres e Adolescentes: Norma Técnica; 126pp.

\section{(DOI INEXISTENTE)}

Murphy, A. \& Hine, B. (2018) Investigating the demographic and attitudinal predictors of rape myth acceptance in U.K. Police officers: developing an evidence-base for training and professional development. Psychology, Crime \& Law, 1-21.(**) https://doi. org/10.1080/1068316X.2018.1503663

Oehme, K., Prost, S. G., \& Saunders, D. G. (2016). Police Responses to Cases of Officer-Involved Domestic Violence: The Effects of a Brief Web-Based Training. Policing, 10(4), 391-407. https://doi.org/10.1093/police/paw039

Page, A. D. (2010). True colors: Police officers and rape myth acceptance. Feminst Criminology, 5, 315334. https://doi.org/10.1177/1557085110384108

Pasinato, W. (2012) Acesso à justiça e violência contra a mulher em Belo Horizonte. São Paulo: Annablume, 232 p. (DOI INEXISTENTE)

Patterson, G. T. (2004). Evaluating the effects of child abuse training on the attitudes, knowledge, and skills of police recruits. Research on Social Work Practice, 14, 273-280. https://doi. org/10.1177/1049731503262390

Porter, R. (1992). Estupro - Será que ele tem um significado histórico? In: S. Tomaselli., \& R. Porter (Eds.), Estupro (pp. 207-227). Rio de Janeiro, RJ. (DOI INEXISTENTE)

Powell, M. B. \& Cauchi, R. (2011) Victims' perceptions of a new model of sexual assault investigation adopted by Victoria Police. Police Practice and Research, 14(3), 228-241 https://doi.org/10.1080/15614263.2011.641376

Powell, M. B. (2008). Designing Effective Training Programs for Investigative Interviewers of Children. Current Issues in Criminal Justice, 20(2), 189-208. https://doi.org/10.1080/10345329.2008.12035804

Resende T. C., Urzedo, R. F., Oliveira, D. M., Segundo, E. M. De O., \& Jorge, M. T._(2011). Aspectos Referentes às Vítimas de Estupro Segundo Processos-Crime em Uberlândia-MG. Revista Fato \& Versões, 5, 43-69. (DOI INEXISTENTE)

Rich, K. \& Seffrin, P. (2013). Police officers' collaboration with rape victim advocates: barriers and facilitators, Violence and Victims, 28(4):681-96. https://doi. org/10.1891/0886-6708.VV-D-12-00044

Scarpati, A. S. (2013). Os Mitos de Estupro e a (Im) Parcialidade Jurídica: A Percepção de Estudantes de Direito Sobre Mulheres Vitimas de Violência Sexual. Dissertação de Mestrado. Programa de Pós-Graduação em Psicologia. Universidade Federal do Espírito Santo. (DOI INEXISTENTE) 
Scarpati, A. S., \& Pina, A. (2017). On national and cultural boundaries: A cross-cultural approach to sexual violence perpetration in Brazil and the United Kingdom. Journal of Sexual Aggression, 1-16. https:// doi.org/10.1080/13552600.2017.1351265

Scarpati, A. S.; Guerra, V. M. \& Bonfim-Duarte, C. N. (2014). Adaptaçāo da Escala de Aceitaçāo dos Mitos de Estupro: Evidências de validade para estudantes de Direito. Avaliaçāo Psicológica (online), 13, 57-65.

\section{(DOI INEXISTENTE)}

Secretaria de Estado da Saúde do Paraná (2015). Protocolo para o atendimento às pessoas em situação de violência sexual / SAS. Curitiba: Superintendência de Atenção à Saúde, 17pp. (DOI INEXISTENTE)

Secretaria de Estado da Saúde do Paraná (2018). Protocolo para o atendimento às pessoas em situação de violência sexual / SAS. Curitiba: Superintendência de Atenção à Saúde, 2ed, 223pp. (DOI INEXISTENTE)

Senado Federal (2018). Observatório da Mulher Contra a Violência. Panorama da violência contra as mulheres no Brasil indicadores Nacionais e estaduais. Brasília, 2ed, 39pp. (DOI INEXISTENTE)

Silva, B. C. da (2017). O atendimento das mulheres vitimas de violência sexual pela segurança pública no Distrito Federal e a percepção dos profissionais da área sobre esse atendimento. Dissertação de Mestrado, Universidade de Brasilia. (DOI INEXISTENTE)

Sleath, E., \& Bull, R. (2012). Comparing rape victim and perpetrator blaming in a police officer sample: Differences between police officers with and without special training. Criminal Justice and Behavior, 39 , 646-665. https://doi.org/10.1177/0093854811434696

Souza, C. M., \& Adesse, L. (2005). Violencia sexual no Brasil: Perspectivas e desafios. Brasília: Secretaria Especial de Políticas para as Mulheres. Brasilia, DF: Secretária Especial de Políticas para as Mulheres: Ipas, 186 p. (DOI INEXISTENTE)

Taylor, S. C., \& Gassner, L. (2010). Stemming the flow: challenges for policing adult sexual assault with regard to attrition rates and under-reporting of sexual offences. Police Practice and Research, 11(3), 240-255. https://doi.org/10.1080/15614260902830153

Temkin, J. (1999). Reporting a rape in London: A qualitative study. Howard Journal of Criminal Justice, 38 , 17-41. https://doi.org/10.1111/1468-2311.00114

Tidmarsh, P., Powell, P., \& Darwinkel, E. (2012). "Whole story": A new framework for conducting investigative interviews about sexual assault. Investigative Interviewing: Research and Practice, 4(2), 33-44. (DOI INEXISTENTE)

UNICEF, UN Women, UNFPA, ILO \& OSRSG/VAC. (2013). Breaking the silence on violence against indigenous girls, adolescents and young women: A call to action based on an overview of existing evidence from Africa, Asia Pacific and Latin America. New York: UNICEF. (DOI INEXISTENTE)
Venema, R. M. (2016). Making Judgements: How Blame Mediates the Influence of Rape Myth Acceptance in Police Response to Sexual Assault. Journal of Interpersonal Violence. https://doi. org/10.1177/0886260516662437

Venema, R. M. (2018). Police Officers' Rape Myth Acceptance: Examining the Role of Officer Characteristics, Estimates of False Reporting, and Social Desirability Bias. Violence and Victim, 33, 176-200. https://doi.org/10.1891/0886-6708.33.1.176

Venema, R. M., Lorenz, K., \& Sweda, N. (2019). A descriptive study of training and its perceived helpfulness among Illinois sexual assault investigators. Journal of Crime and Justice, 1-15. https:// doi. org/10.1080/0735648X.2019.1601585

Zoltowski, A. P. C., Costa, A. B., Teixeira, M. A. P., \& Koller, S. H. (2014). Qualidade Metodológica das Revisões Sistemáticas em Periódicos de Psicologia Brasileiros. Psicologia: Teoria e Pesquisa, 30, 97-104. https://doi.org/10.1590/S0102-37722014000100012

\section{Endereço para correspondência}

Arielle Sagrillo Scarpati (Rua Lumberto de Azevedo Maciel, Jardim Camburi - Vitória, ES, 29090-700; 027 99757-9727; arielle.psicologia@gmail.com)

\section{Autor I}

Nome: Arielle Sagrillo Scarpati

Titulação Acadêmica: Doutorado em Psicologia Forense

Afiliação Institucional: Universidade Federal do Rio Grande do Sul (UFRGS).

\section{Autor II}

Nome: Silvia Helena Koller

Titulação Acadêmica: Doutorado e Pós-Doutorado em Psicologia

Afiliação Institucional: Universidade Federal do Rio Grande do Sul (UFRGS). 
TABELA 01 - Trabalhos incluídos na revisão sistemática de literatura

\begin{tabular}{|c|c|c|c|}
\hline Artigo & Objetivos & Método & $\begin{array}{l}\text { Metodologia do Treinamento } \\
\text { Proposto e/ou Avaliado }\end{array}$ \\
\hline 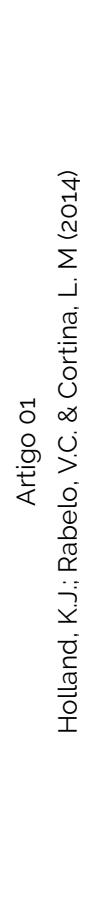 & $\begin{array}{l}\text { Avaliar o poder pre- } \\
\text { ditivo de treinamen- } \\
\text { to sobre violência } \\
\text { sexual no que se } \\
\text { refere ao nivel de } \\
\text { conhecimento do } \\
\text { tema, e explorar } \\
\text { a relação entre } \\
\text { fatores institucionais } \\
\text { (ramo de serviço, } \\
\text { classificação) e } \\
\text { individuais (gênero, } \\
\text { histórico de agres- } \\
\text { são) e percepções } \\
\text { de eficácia do } \\
\text { treinamento. }\end{array}$ & $\begin{array}{l}\text { Análise secundária dos dados } \\
\text { gerados pela "Pesquisa de } \\
\text { Trabalho e Relações de Gênero } \\
\text { dos Membros do Active Duty } \\
\text { de } 2010 \text { " (WGRA); Participantes: } \\
26.505 \text { (N = 15.859 homens; } \\
59,8 \% \text { e N = } 10.646 \text { mulheres; } \\
\text { 40,2\%) militares, distribuidos en- } \\
\text { tre aqueles que haviam recebido } \\
\text { ou não (1 = Não e } 2 \text { = Sim) treina- } \\
\text { mento em tópicos relacionados } \\
\text { à agressão sexual nos últimos } 12 \\
\text { meses. Instrumentos: questões } \\
\text { abertas e fechadas sobre o trei- } \\
\text { namento recebido (ex. "O curso } \\
\text { fornece uma boa compreensão } \\
\text { de quais ações são considera- } \\
\text { das uma violência sexual?") e a } \\
\text { percepção quanto à eficácia do } \\
\text { treinamento ("Na sua opinião, } \\
\text { quão eficaz foi o treinamento } \\
\text { que você recebeu em..."). }\end{array}$ & $\begin{array}{l}\text { Treinamento com base em } \\
\text { evidências científica. Por } \\
\text { ser adaptado ao ambiente } \\
\text { no qual é ofertado, leva } \\
\text { em consideração aspectos } \\
\text { como o gênero, a cultura e } \\
\text { as crenças do público-alvo. } \\
\text { As atividades são ministradas } \\
\text { por profissionais treinados, a } \\
\text { partir de um formato intera- } \\
\text { tivo; intercalando palestras, } \\
\text { discussões, dramatizações e } \\
\text { exercícios. Tem por objetivo, } \\
\text { transmitir informações acerca } \\
\text { a) das formas pelas quais } \\
\text { "espectadores da violência" } \\
\text { (bystanders) podem intervir, b) } \\
\text { empatia, c) consentimento, d) } \\
\text { comportamentos aceitáveis } \\
\text { neste contexto, e e) a impor- } \\
\text { tância de relacionamentos } \\
\text { saudáveis. }\end{array}$ \\
\hline
\end{tabular}

Não são oferecidas informações a respeito dos protocolos de treinamento. Infere-se, apenas, e com base nas respostas obtidas, que os treinamentos são oferecidos presencialmente e/ou on-line, e abordam temais tais quais: técnicas para interrogar suspeitos, questões legais sobre a prisão, interação com a vitima, motivações do agressor, treinamento de empatia, álcool/droga em contexto de agressões sexuais, aceitação do Mito de Estupro (RMA), dentro outros. A maioria (78\%; $n=169$ ) dos investigadores relataram uma média de $76.9 \mathrm{~h}$ de treinamento em sala de aula $(D P=57,1)$.

Aproximadamente $61 \%(n=$ 131) dos investigadores que receberam treinamento em sala de aula indicaram, ainda, terem recebido algum tipo de acompanhamento posterior $(M=107,5 h, D P=122,8)$.
Participantes que classificaram o treinamento como compreensivo e suficiente, demonstraram uma precisão significativamente maior $(p<.001)$ em seu conhecimento de recursos e protocolos oficiais quando comparado aos grupos de menor ou nenhuma exposição. Também foi observado um efeito de gênero e histórico de violência, com participantes do sexo masculino e sem histórico de violência sexual considerando o treinamento mais eficaz na redução/prevenção destas violações do que homens e mulheres sobreviventes de violência. O mesmo resultado foi encontrado para as mulheres que não haviam sido vitimas, quando comparadas com homens e mulheres sobreviventes. De modo geral, independentemente do histórico de vitimização, mulheres perceberam que o treinamento era menos efetivo que os homens.

Resultados indicam que muitos dos temas considerados importantes para o trato de vitimas de violência sexual (ex., RMA e interação com vitimas que sofrem de PTSD ou outros sintomas de trauma) não têm sido ofertados adequadamente a estes profissionais. Isto porque ainda há, de acordo com os autores, maior ênfase em orientações legais, com foco nas "hard skills" (ex. técnicas de entrevista) em detrimento das chamadas "soft skills" (ex. empatia e habilidades para criação e manutenção de vínculo com a vítima). Participantes também indicaram que treinamentos adicionais e continuados poderiam levar a melhores investigações de agressão sexual; demonstrando especial interesse no que se refere a temas como a identificação e preservação de evidências em casos de agressão e técnicas de entrevista que incluam informações sobre empatia. compaixão e respeito, bem como a compreensão da neurobiologia do trauma e cultura do estupro; de modo a reduzir ou evitar a revitimização. 


\begin{tabular}{|c|c|c|c|c|}
\hline Artigo & Objetivos & Método & $\begin{array}{l}\text { Metodologia do Treinamento } \\
\text { Proposto e/ou Avaliado }\end{array}$ & Resultados \\
\hline 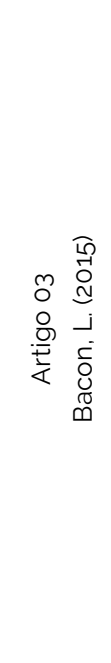 & $\begin{array}{l}\text { Apresentar os } \\
\text { esforços realizados } \\
\text { Polícia Nacional da } \\
\text { Libéria (LNP) entre } \\
\text { os anos de } 2003 \text { e } \\
2013 \text { para: a) recru- } \\
\text { tar mais oficiais do } \\
\text { sexo feminino e b) } \\
\text { treinar uma unidade } \\
\text { especializada para } \\
\text { abordar a violência } \\
\text { sexual e de gênero. }\end{array}$ & Estudo de Caso & $\begin{array}{l}\text { O programa oferecido aos } \\
\text { profissionais lotados na } \\
\text { seção dedicada a responder } \\
\text { a denúncias de violência } \\
\text { doméstica, agressão e crimes } \\
\text { contra crianças não é descrito. } \\
\text { Entretanto, as informações } \\
\text { disponibilizadas indicam que o } \\
\text { mesmo foi ofertado em turmas } \\
\text { de } 25 \text { oficiais e teve como foco } \\
\text { os seguintes tópicos: a criação } \\
\text { de relatórios investigativos, } \\
\text { coleta de evidências e manu- } \\
\text { tenção de confidencialidade } \\
\text { em casos de denúncias de vio- } \\
\text { lência doméstica/ sexual. }\end{array}$ & $\begin{array}{l}\text { Coletivamente, os resultados reporta- } \\
\text { dos indicam que as avaliações externas } \\
\text { ao programa criado são mistas. Isto por- } \\
\text { que, apesar de um aparente aumento } \\
\text { no número de casos reportados, não } \\
\text { há informações suficientes para que se } \\
\text { afirme a efetividade do modelo criado. } \\
\text { Deste modo, a autora conclui indicando } \\
\text { a necessidade de uma investigação } \\
\text { mais aprofundada do mesmo. }\end{array}$ \\
\hline 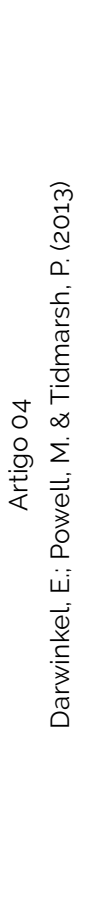 & $\begin{array}{l}\text { Revisitar o traba- } \\
\text { lho realizado por } \\
\text { Lonsway et al. (2001) } \\
\text { e Patterson (2004) } \\
\text { e testar a eficácia } \\
\text { de um programa de } \\
\text { treinamento obriga- } \\
\text { tório projetado para } \\
\text { policiais especia- } \\
\text { lizados em abuso } \\
\text { sexual (infantil e } \\
\text { adulto). }\end{array}$ & $\begin{array}{l}\text { Participantes: } 77 \text { oficiais da Polí- } \\
\text { cia de Victoria, Austrália; sendo } \\
\text { composta por } 66 \% \text { destes do } \\
\text { sexo masculino e } 34 \% \text { do sexo } \\
\text { feminino. Desenho do estudo: } \\
\text { pré e pós-teste. Instrumen- } \\
\text { to: questionário contendo } 12 \\
\text { cenários de violência, seguidos } \\
\text { de duas perguntas sobre o caso } \\
\text { (O quão confiante você se sente } \\
\text { confiante de que o caso será jul- } \\
\text { gado? "Quanta responsabilidade } \\
\text { deve ser atribuida à vítima neste } \\
\text { cenário?"). Em seguida, foram } \\
\text { solicitados a justificarem suas } \\
\text { respostas, listando até cinco } \\
\text { fatores (em ordem de impor- } \\
\text { tância) que eles consideravam } \\
\text { durante a tomada de decisões. }\end{array}$ & $\begin{array}{l}\text { É baseado na abordagem } \\
\text { "Whole Story" para a inves- } \\
\text { tigação de crimes sexuais } \\
\text { e tem duração de quatro } \\
\text { semanas. É conduzido por } \\
\text { profissionais treinados e eu } \\
\text { conteúdo centra-se na dinâ- } \\
\text { mica da violência e técnicas } \\
\text { de entrevista. Mais especifi- } \\
\text { camente, o curso ministrado } \\
\text { inclui os seguintes conteúdos, } \\
\text { e na seguinte ordem: modelo } \\
\text { introdutório, compreensão do } \\
\text { comportamento das vitimas } \\
\text { e dos agressores, discussão } \\
\text { de casos e, por fim, técnicas } \\
\text { de entrevista (teoria e prática). } \\
\text { Participantes também são } \\
\text { solicitados a demonstrar seus } \\
\text { conhecimentos e habilidades } \\
\text { de entrevista em diversas situ- } \\
\text { ações interativas e role-plays. }\end{array}$ & $\begin{array}{l}\text { Coletivamente, foram observadas } \\
\text { mudanças nos niveis de confiança no } \\
\text { relato da vítima e na responsabilidade } \\
\text { atribuida à mesma. Mais precisamen- } \\
\text { te, o padrão de resultados sugere } \\
\text { que a confiança no relato da vítima } \\
\text { aumentou (do pré para o pós-treina- } \\
\text { mento), enquanto a percepção de sua } \\
\text { "responsabilidade" diminuiu. Resulta- } \\
\text { dos apresentados também oferecem } \\
\text { evidência qualitativa para justificar } \\
\text { a importância de um treinamento } \\
\text { focado na compreensão da dinâmica } \\
\text { da violência sexual no processo de } \\
\text { denúncia e condução do caso. }\end{array}$ \\
\hline
\end{tabular}

